\title{
The aim and philosophy of patient monitoring
}

\author{
J. S. S. STEWART \\ Royal Albert Edward Infirmary, Wigan
}

\begin{abstract}
Summary
The history of monitoring is traced from ancient times until the invention of transducers and computers. The relevance of progress in resuscitation is emphasized.

The more recent evolution of electromedical apparatus is considered from single signal detection, display and alarm to multiple signal processing, trend analysis and diagnosis.

The aim of patient monitoring is to give warning of early or dangerous deterioration and to achieve this by obtaining an optimal compromise involving many design factors, clinical, engineering and economic.

A new philosophy is illustrated by the specification and development of the Lifeline patient monitor. The translation of clinical diagnoses into electronic switching logic is of particular importance.
\end{abstract}

AT FIRST sight it might appear not only impertinent for a clinician to speak about engineering but also pretentious to deal with philosophy. In fact this is only a superficial examination of the latter subjects, little more than an attempt to grapple with an outstanding problem of our age, to establish communication. Thus it will be appreciated that this exploratory excursion is based on little knowledge and far less understanding. It is the simple story of a new approach to patient monitoring.

\section{History of monitoring}

The development of patient monitoring is closely linked to that of resuscitation. A common aim is the welfare and treatment of patients and it is logical to progress from clinical resuscitation to the early detection or prevention of clinical catastrophes. The principal techniques of resuscitation are expired air ventilation and external cardiac massage. Surprisingly it does not appear to have been pointed out before that there is a description of expired air ventilation which is as old as the Bible. It was Jean Astruc, a physician from Montpellier, who first presented evidence that Genesis is of composite origin and authorship; the same stories are sometimes told by different authors (Carpenter, 1948). The oldest story is that of the creation and in the second version of this the creation of man is described (Genesis 2, 7):
'And the Lord God formed man of the dust of the ground and breathed into his nostrils the breath of life; and man became a living soul.'

This description of expired air resuscitation could hardly be more modern for it is now recognized that mouth-to-nose ventilation is better than mouth-tomouth. It came too soon for the electronic age but not so with external cardiac massage. The recent description of external massage was not in fact the first because, as Jude, Kouwenhoven \& Knickerbocker (1964) point out, successful resuscitation using this technique was reported by Koenig in 1885. The method was forgotten but was rediscovered, quite independently, by Kouwenhoven, Jude \& Knickerbocker (1960). This new description of the technique and the interest in resuscitation which subsequently burgeoned has been an important stimulus to the development of patient monitoring.

The earliest written record relevant to the history of patient monitoring is contained in the papyrus discovered by Ebers (1875). This remarkable document, written in $1550 \mathrm{BC}$, makes it clear that the ancient Egyptian physicians were familiar with the fact that the peripheral pulse could be correlated with the heart beat:

'As to faintness of the heart. It is that the heart does speak or the vessels of the heart are dumb, there being no perception under the fingers.'

The next contribution of importance was made some 3000 years later when Galileo discovered the principle of the pendulum in 1658 and used it to measure the pulse rate (Graham, 1956). This was perhaps the first example of clinical measurement. The medical electronic age began when Waller (1887) recorded the electrical activity of the human heart. Further notable contributions to the science of clinical measurement in the assessment of a patient's condition were made by MacKenzie (1925), a general practitioner cardiologist from Burnley, who emphasized the importance of graphical records of the pulse rate and blood pressure.

The foundation of the computer age was laid by Boole (1854) who developed the fundamental principles of symbolical reasoning in his monograph on the laws of thought. Although the importance of 
this work was long neglected its application was advanced by translation of Boolean algebra into switching logic. Practical exploitation and technological development were not long delayed and ENIAC, the first electronic digital computer, was operating in 1945.

The more recent evolution of monitoring equipment has been greatly accelerated by the technological resources of the electronics age and has largely taken place within the last decade. In the previous decade, however, the continuous oscilloscope display of the electrocardiogram was widely used during and after cardiac surgery and latterly high and low alarms were incorporated in these monitors. Later the vital signs, heart rate, blood pressure and respiratory rate were monitored (Geddes et al., 1962). Computers were used to analyse the data (Freiman \& Steinberg, 1964) and facilities for 'on-line' computing were developed (Jensen et al., 1966). False alarms have always been a problem but are greatly reduced in frequency if an alarm compromise is adopted (Raison et al., 1968) in which an alarm is given only if two parameters are abnormal. The present Lifeline monitor, which is based on logical analysis of vital signs, is the latest stage in the evolution of monitoring equipment. The sequence is summarized in Table 1 .

TABLE 1. The recent evolution of monitoring equipment

Continuous electrocardiogram display

High and low alarms

Vital signs monitor: HR, BP, RR.

Computer processing of data

On-line computing

Alarm compromise

Lifeline monitor and diagnostic computer

\section{Aim, philosophy and specification}

Originally a monitor was a person who admonished others but now the meaning has slightly changed. A monitor is a thing which gives a warning. The aim of patient monitoring is, by continuous surveillance, to detect early or dangerous deterioration, with reliability and accuracy, and to give an appropriate warning or alarm. The functional parts of such a monitor are concerned with input, data handling and output. These are called sensors, processor and communicator. It is important that the surveillance should be continuous, the processing immediate, the diagnosis accurate and the warning early enough to be clinically useful. The general trend in electronics is towards complexity and sophistication but the need in clinical practice is for a reliable, robust and relatively inexpensive machine. Reduction in the cost of monitoring is essential if facilities are to be provided for the many patients who could benefit. Thus philosophy must be tempered with practicality. The importance of useful inexpensive equipment was emphasized by Taylor (1968) who cited the annual report of the Chief Medical Officer at the Ministry of Health:

'New equipment should demonstrate real value to the patient, the staff, or the service. It must also be provided at a price that allows it to compete with other needs within the financial resources available.'

and the same view is emphasized by Wolff (1968) who believes that

'.. there should be a deliberate concentration on those projects where technology acts as the leveller in making good care available to the many rather than performing miracles for the few.'

From the purely technological viewpoint clear and precise requirements have been laid down by Fisher (1968). These are quoted, with minor paraphrasing, as follows:

'Physiological signals must still be evaluated and correlated by the observer in order to assess the immediate condition of any patient and the deduction of impending crises in many acute situations relies heavily on the intuitive skill and experience of the clinician.'

'Early detection and identification of significant deterioration in a patient's condition must be achieved "on-line" to the computer so that data may be presented to the observer in an immediate and intelligible form requiring minimal visual interpretation if it is to be of real benefit in emergency situations.'

Just such a machine, the Lifeline patient monitor and diagnostic computer, has been constructed and it is appropriate to consider some of its specifications which translate a philosophy into reality. Essentially it is the product of an optimal compromise involving many design factors; clinical, engineering and economic. The new machine does make a diagnosis, and it meets precisely the requirements for 'on-line' monitoring of automatic analysis, computing and output with alarm of cardiac arrest, hypoxia or shock, with early warning of tachycardia, bradycardia, hypotension or cyanosis and with warning of sensor or power failure.

The principal features can be further specified in respect of sensors, signal analysis and communication. The analyser unit should be usable with a wide variety of sensors; skin contact, intra-arterial or other types. Three sensors, related respectively to heart action, circulation and oxygenation, are used. Thus input to the first may, for example, be heart or pulse rate or cardiac output; to the second: blood pressure, flow, velocity or volume; to the third: respiration rate, skin colour, blood or tissue oxygen, electroencephalogram. The clinician tunes each channel to the individual patient and sets the range which is appropriate. In doing this he draws upon his clinical 
experience and exercises his skill. The level of output from each sensor is categorized as 'high', 'normal' or 'low' according to the chosen clinical setting. These signals are processed by the logic unit in accordance with previously defined clinical logic (Stewart, 1969a). Signal processing and surveillance is continuous, with accurate diagnosis in spite of the adaptability of the logic to accommodate a wide variety of sensors. The displayed output indicates the presence of an abnormality, clinical or sensor, and shows at a glance not only the diagnosis but also the features of which the diagnosis is compounded. Early warning is given if the signal from any one sensor is abnormal and urgent alarm is given if the combination of abnormal signals is dangerous. Warnings are also given in the event of sensor or power failure. It is equally important to define what a monitor should not do and display of oscilloscope wave form and metered absolute values are dispensed with as are all print out and other records. Although arrhythmia detection would be a valuable adjunct it is not included in the present monitor.

\section{Evolution of Lifeline}

From a prior interest in resuscitation (Stewart, 1965) it was clear that circulatory arrest may be due to primary cardiac arrest or to acute circulatory failure commonly due to shock or hypoxia. A small epidemiological study of deaths from myocardial infarction (Stewart \& Bardhan, 1966) emphasized the very obvious fact that results are poor if resuscitation is not attempted. It was not attempted in many cases because the emergency was not diagnosed soon enough. The outstanding need was for an efficient cheap monitor.

Anaesthetists, physicians and surgeons are in the main, but not of course exclusively, concerned about, respectively, respiration, heart and circulation, that is with the early detection, avoidance or prevention of hypoxia, cardiac arrest and oligaemic shock. A monitor should be able to detect all these emergencies and to give an early warning of arrest. A monitoring system based solely on the electrocardiogram will frequently fail to diagnose hypoxia before irreversible brain damage has already occurred. Thus at least one more parameter must be used. Obviously reliability will be increased if the diagnosis is based on simultaneous abnormality of two parameters. The addition of a third parameter contributes a large and quite disproportionate advantage, for the diagnosis of each of these important conditions, arrest, hypoxia, shock, can then be based on the presence of simultaneous abnormalities in at least two parameters. With three parameters and three levels (high, normal, low) for each there are twenty-seven permutations. This set of twenty-seven forms the basis of a truth table and to each possibility was assigned a clinical meaning in respect both of diagnosis and urgency(Stewart, 1969a). Preliminary specifications of a patient monitor and diagnostic computer using the logic of the truth table were drawn up at the same time. The logic unit is the electronic switching system which enables the computer to make a diagnosis. A prototype was constructed in the Department of Engineering Design and Production (Sadera et al., 1969) and the logic unit proved satisfactory in animal experiments (Levy et al., 1969).

A useful introduction to switching logic has been given by Lockwood (1968 a and b). There are three basic types of gate which permit one-way transmission of a signal according to the function OR, AND or NOT of the gate. An OR gate gives an output signal if there is a signal in one or other input. An AND gate gives an output signal if there is a signal in one AND the other input. The AND gate with two inputs is also called a 2 gate. A NOT gate gives an output signal if there is NOT a signal in the input. The functions NOT OR and NOT AND are combined in NOR and NAND gates.

The process of translation from clinical to electronic logic is of particular importance. Consideration of the pulse rate and blood pressure during the development of oligaemic shock may be used to exemplify, in very much simplified form, the translation process. The pulse rate rises progressively to a peak and then rapidly declines. The blood pressure is steady at first but later rises due to overcompensation of physiological mechanisms and then declines (Fig. 1). The corresponding electronic logic diagram (Fig. 2) shows how OR gates and AND gates are used to make a diagnosis. An analogue signal corresponding to heart rate (HR) passes through a level comparator which categorizes the signal as high $(+)$ or low (-). An early warning signal ABNORMAL is given via an OR gate if heart rate is high OR low, OR if blood pressure (BP) is high OR low. A wARNING is given if heart rate is high AND blood pressure is high. An

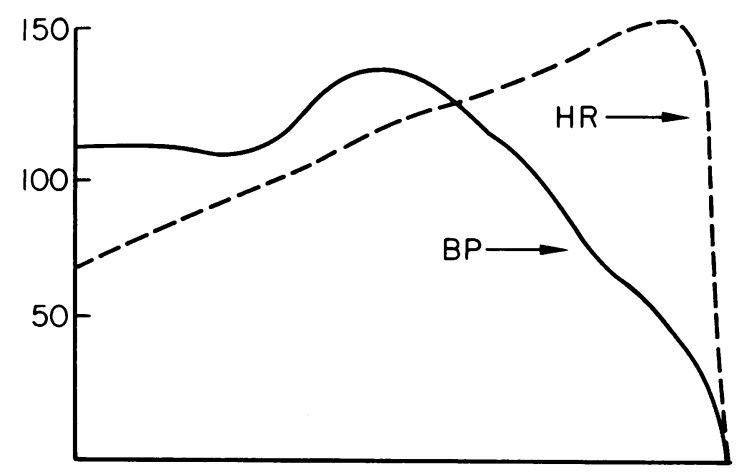

FIG. 1. Diagrammatic representation of shock, showing heart rate and blood pressure changes with time. The stage of hypertension does not always occur. 


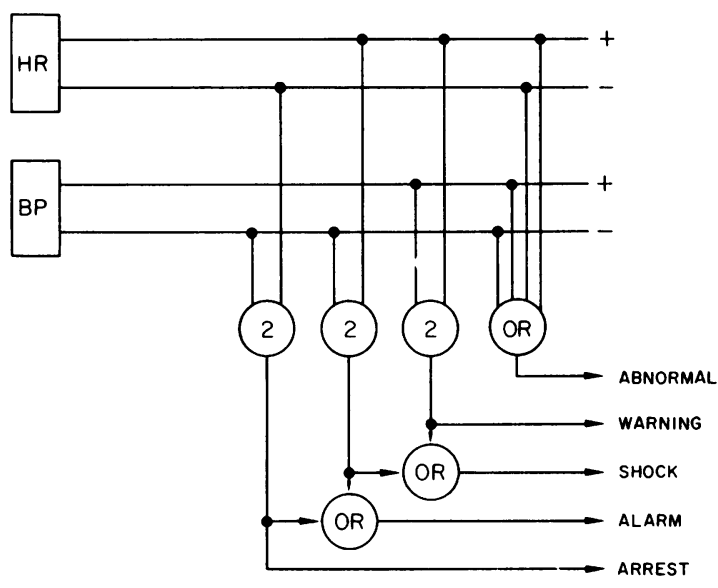

Fig. 2. Translation of clinical state by electronic switching logic into graded warning and diagnosis.

ALARM is given if heart rate is high AND blood pressure is low. If the output from one oR other of these two combinations is positive a diagnosis of SHOCK is given. This very simple example shows how different signals or combinations of signals give an early warning, warning and alarm together with the appropriate diagnosis. The logic unit was the subject of a patent application by the author in 1968. The rights are being assigned to a trust in which the author has no financial interest and world-wide patent protection has been sought.

The role of inventor might at first appear strange for a clinician but Eiloart (1969) defines the qualities thus:

'A good inventor is generally not compatible with industry, especially with large scale industry. Such a man must be a self-confident enthusiast. He is likely to work very hard and he automatically questions accepted practice. He will also need to be a flexible and an energetic self-starter. Expressed extremely these virtues may tend towards egotism, obsession, wilfulness, volatility and eccentricity.'

What surgeon does not recognize these traits in his physician colleagues and what physician does not diagnose them in every surgeon? Inventions by hospital service employees was the title of a hospital memorandum (HM(67)63) asking Boards and Committees to keep the Ministry of Health informed of all inventions which hospital service employees have made, or helped to make. Taylor (1968) has emphasized that the Department is taking an increasing part in the development of equipment and funds are available to assist the clinician to do this. The Department of Health and Social Security, as it now is, kindly provided a grant for the development of a solid state version of the Lifeline monitor. The prototype logic unit, which was demonstrated at the
International Inventor's Exhibition at Brussels in March, 1969, was awarded a gold medal and the jury's felicitations (Report, 1969). The development was briefly described (Stewart, 1969b) and the solid state version, which was constructed under the supervision of Dr J. R. B. Greer, is being described by Greer, Greig, McLintock \& Stewart (1969). After vicissitudes, which Greer (1970) describes at this Symposium, the solid state version was recently delivered to the Department of Health for testing. Preliminary clinical trials indicate that there will be few false alarms but more sensor faults since three sensors are used. It is probable, however, that the vast majority of these can be corrected without distressing the patient. The Lifeline monitor has been developed in the hope that it will bring monitoring of increased efficiency to more patients with less distress. Initial experience suggests that it is worthy of further trial.

\section{References}

Boole, G. (1854) An investigation of the laws of thought on which are founded the mathematical theories of logic and probabilities. Dover. New York.

Carpenter, J.E. (1948) Introduction to the Pentateuch, in Peake, A.S. (1948). A Commentary on the Bible. Nelson, London.

Ebers, G. (1875) Papyrus Ebers. Leipzig.

EILOART, T.M.B.(1969) Seeking and developing new products and processes. The Inventor, 9, (1), 14.

Fisher, B.J. (1968) 'On line' system for patients has a double advantage. Electronics Weekly, October 30.

Freiman, A.H. \& Steinberg, C.A. (1964). The analysis of simultaneously recorded cardiovascular data with the digital computer. Annals of the New York Academy of Sciences, 115, 1091.

Geddes, L.A., Hoff, H.E., Spencer, W.A. \& Vablona, C. (1962) Acquisition of physiological data at the bedside: a progress report. American Journal of Medical Electronics, $1,62$.

GrahaM, H. (1956) Surgeons All, p. 357. Rich \& Cowan, London.

Greer, J.R.B. (1970) A clinical computer and monitor. Postgraduate Medical Journal, 46, 369.

GreER, J.R.B., Greig, E.W., McLintock, I.J. \& SteWART, J.S.S. (1970) The Lifeline patient monitor. (In preparation).

Jensen, R.E., Shubin, H., Meagher, P.F. \& Weil, M.H. (1966) On-line computer monitoring of the seriously ill patient. Medical and Biological Engineering, 4, 265.

JUDE, J.R., KOUWENHOVEN, W.B. \& KNICKERBOCKER, G.G. (1964) External cardiac resuscitation. Monographs on the Surgical Sciences, 1, 1.

KoUWENHOVEN, W.B., JUDE, J.R., KNICKERBOCKER, G.G. (1960) Closed chest cardiac massage. Journal of the American Medical Association, 173, 1064.

LeVY, L.S., LeE, W.R., GuRuswamy, B.A. \& STEWART, J.S.S. (1970) Experimental studies with a clinical monitor and diagnostic computer. Postgraduate Medical Journal, 46, 366.

Lockwood, F.B. (1968a) Logic switching circuits. Machinery and Production Engineering, 112, 249.

Lockwood, F.B. (1968b) Logic systems. Machinery and Production Engineering, 112, 986.

MacKenzIE, J. (1925) Diseases of the Heart. Oxford University Press. 
Raison, J.C.A., Beaumont, J.O., Russell, J.A.G., Osborn, J.J. \& Gerbode, F. (1968) Alarms in an intensive care unit: an interim compromise. Computers and Biomedical Research, 1, 556.

REPORT (1969) Brussels exhibition 1969. The Inventor, 9, 26.

Sadera, T.D., Guruswamy, B.A., Penny, R.K., Stewart J.S.S. (1969) Design of a circulatory failure analyser. (In press.)

STEWART, J.S.S. (1965) Advances in the management of cardiac arrest. Journal of the Royal College of Surgeons (Edinburgh), 10, 85.

Stewart, J.S.S. (1969a) Meaningful monitoring. Lancet, i, 1305.
Stewart, J.S.S. (1969b) A clinical diagnostic computer and monitor. European Surgical Research, 1, 248.

STEWART, J.S.S. \& BARDHAN, K.D. (1966) Intensive care units for myocardial infarction. British Medical Journal, i, 1297.

TAYLOR, D.J.W. (1968) The medical engineering interface in Britain. Instrumentation in Medicine. Proceedings of Second European Symposium on medical electronics, London 1967. Hanover Press, London.

WALLER, A.D. (1887) A demonstration on man of electromotive changes accompanying the heart's beat. Journal of Physiology, 8, 229.

WOLFF, H.S. (1968) Bioengineering-the next few years. Bio-Medical Engineering, 3, 15.

\title{
Sensors
}

\author{
H. S. WOLFF \\ Medical Research Council, Hampstead Laboratories, Holly Hill, London
}

\section{(Abstract)}

ANY CHANNEL of a monitoring system, however simple, must consist at least of a sensor or transducer, a signal processor and a display device. Of these three components, signal processing which is usually a purely electronic function presents the smallest problems, display devices are adequate at the present state of the art, but the acquisition of the input signal can still present considerable difficulties.

These difficulties can be classified under three headings. First, the choice of the signal to be sensed. For reasons of patient comfort, convenience and safety, signals are often chosen which may only be remotely connected to the physiological process which is to be monitored. An example is the indirect measurement of arterial blood pressure, which however good the sensing system may be, will always be inherently more unreliable than direct measurement. Second, the stability of the transducer itself, problems of calibration and sterilization and resistance to maltreatment and overload. Thirdly, methods of attachment or connection to the patient such that voluntary and involuntary movements cause the minimum of artifact and present the least obstacle to nursing procedure.

Sensors in present day use and some which are under development were discussed with reference to the above classifications. 